\title{
FOULQUIÉ-RUBIO, A. I., VARGAS-URPI, M. Y FERNÁNDEZ PÉREZ, M. (EDS.) (2018). PANORAMA DE LA TRADUCCIÓN Y LA INTERPRETACIÓN EN LOS SERVICIOS PÚBLICOS ESPAÑOLES: UNA DÉCADA DE CAMBIOS, RETOS Y OPORTUNIDADES. N ${ }^{\mathrm{O}}$ PÁGINAS: 231. EDITORIAL: COMARES. ISBN: 978-84-9045-672-9.
}

\author{
Reseñado por Abdallah Jamal Albeetar \\ Universidad de Granada \\ abdjamalbi4@gmail.com
}

El objetivo de esta obra es actualizar la descripción de la situación en España de la Traducción e Interpretación en los Servicios Públicos (en adelante, TISP) después de una década convulsa, representada principalmente por la crisis económica, que ha dejado como secuela una mayor precarización en la situación laboral de quienes ejercen como intérpretes y ha impedido alcanzar un nivel de profesionalización. Comunica, Observatorio Permanente sobre Traducción e Interpretación en los Servicios Públicos, pretende, mediante este volumen, reflejar de manera exhaustiva la situación actual de la profesión en España en los diferentes ámbitos: el judicial, el sanitario y el policial hasta el año 2016, teniendo en cuenta la publicación anterior Retos del siglo XXI en comunicación intercultural: nuevo mapa lingüístico y cultural de España, editado por Carmen Valero Garcés y Francisco Raga Gimeno en 2006. Con este objetivo, el libro expone la situación de la TISP en diferentes comunidades autónomas, al amparo de diferentes estudios realizados por los autores que componen los artículos del monográfico.

En la introducción del libro, las editoras del volumen (Ana-Isabel Foulquié-Rubio, Mireia Vargas-Urpi y Magdalena Fernández Pérez) ofrecen un resumen actualizado sobre la TISP en España en diferentes ámbitos. Sostienen que la lectura del mapa actual de la profesión deja una sensación agridulce, pues los recortes y la falta de sensibilización general de la necesidad de dichos servicios han causado una desprofesionalización en los diferentes ámbitos pero, no obstante, existe un gran interés en el ámbito de la investigación, así como la oferta académica en TISP de numerosas universidades españolas.

El monográfico se abre con la descripción de la TISP en Andalucía por parte de Elisa Calvo y Francisco J. Vigier Moreno. Para actualizar la descripción de la TISP en la comunidad autónoma, los autores se basan en artículos, monografías, tesis, trabajos fín de máster y trabajos fin de grado. Los mismos concluyen que la introducción de la interpretación telefónica ha sido probablemente el fenómeno más novedoso en esta década, siendo Andalucía la comunidad autónoma pionera en este sentido, al licitar el servicio de teleinterpretación del Servicio Andaluz de Salud desde hace 15 años.

Almudena Nevado Llopis, por su parte, actualiza los datos relativos a Aragón, que ha experimentado un notable incremento de la población extranjera. Se empleó una metodología mixta basada en el uso de diferentes técnicas como entrevistas semiestructuradas o investigaciones previas. En este sentido, para atender a estos solicitantes de atención lingüística, el gobierno autonómico ha contratado a empresas externas para proporcionar servicios de traducción e interpretación. Como iniciativa esperanzadora, el ámbito sanitario cuenta con la total disponibilidad horaria del servicio de interpretación telefónica, y la contratación de intérpretes cualificados. En cambio, en los sectores judicial y policial, no 
existen criterios de profesionalización para las personas que ejercen de intérpretes. Por último, las combinaciones lingüísticas ofertadas en los planes de estudios de las dos universidades con Traducción e Interpretación presentes en la comunidad autónoma no se corresponden con las necesidades existentes en este territorio.

En Cataluña y en las Islas Baleares, Xus Ugarte Ballester y Mireia Vargas-Urpi describen la situación actual de la TISP mediante los resultados obtenidos a través de entrevistas, encuestas y cuestionarios. Las mismas señalan que en Cataluña no existe un acuerdo para las soluciones adoptadas para resolver los problemas de las barreras lingüísticas dado que son iniciativas de las propias instituciones implicadas, y no por parte de las políticas públicas. Cabe señalar que el ámbito judicial es el único que dispone de un reglamento claro relativo al perfil profesional de los intérpretes. No obstante, en el ámbito policial se recurre a los intérpretes profesionales como último recurso cuando se detectan problemas de comunicación. En Baleares, la situación de la TISP no ha mejorado sustancialmente en los últimos años, debido a la alternancia de gobiernos autonómicos de distinta ideología. El servicio de interpretación telefónica y el servicio de mediación intercultural son los únicos servicios prestados.

Jessica Pérez-Luzardo Díaz y Magdalena Fernández Pérez presentan, mediante cuestionarios, la situación en que se encuentra la provisión de la TISP en el archipiélago canario, destino de millones de turistas procedentes de todo el mundo. Las autoras concluyen su artículo detectando carencias en todos los ámbitos de TISP. Se añade a ello la falta de profesionalización de los intérpretes y la calidad de los servicios prestados, así como la escasa oferta académica en este sentido.

A continuación, Jesús Baigorri Jalón y Críspulo Travieso Rodríguez abordan el capítulo correspondiente a Extremadura, sobre la base de datos obtenidos mediante una encuesta dirigida a diferentes servicios públicos, estadísticas oficiales y noticias de diarios digitales. Se desprende de su estudio que, si bien el número de la población inmigrante ha disminuido, el número de los turistas y, por ende, la demanda de servicios lingüísticos ha aumentado. Se sigue recurriendo todavía a soluciones ad hoc, sin olvidar la incorporación de la empresa Seprotec, que presta servicios de traducción en los ámbitos policial y judicial. Por su parte, la empresa de interpretación telefónica Dualia presta los servicios de interpretación en el ámbito sanitario y en las urgencias del 112.

Maribel del Pozo Triviño y Doris Fernandes del Pozo hablan de la situación de la TISP en Galicia hasta el año 2016, recurriendo a investigaciones previas, la consulta de datos oficiales y el análisis de la legislación y los pliegos de la contratación, para describir la situación actual de la profesión. Su estudio concluye que la situación demográfica y sociolingüística ha cambiado notablemente en Galicia en los últimos diez años, lo que ha creado un nuevo escenario que incluye nuevas lenguas y nuevas realidades sociales. No obstante, la provisión de servicios de TISP continúa siendo la misma. Sin embargo, y como nota positiva, la intensa labor de concienciación de diferentes asociaciones nacionales ha consistido en participar en proyectos para mejorar los servicios de TISP en Galicia.

Por su parte, las comunidades autónomas de Madrid y Castilla La Mancha han sido estudiadas por Carmen Valero Garcés y Sofía Monzón. Los ámbitos estudiados fueron los más representativos en la TISP: el ámbito sanitario y legal. Las autoras empezaron por un estudio demográfico en ambas comunidades autónomas. Su metodología descriptiva y contrastiva se llevó a cabo a través del uso de encuestas presenciales, telefónicas y por internet. Las conclusiones obtenidas del estudio indican que ambas comunidades autónomas conocieron un descenso notable en el número de la población extranjera a causa de la crisis financiera, y, por lo tanto, menos personas alófonas necesitan los servicios de TISP en sus diferentes ámbitos. Las autoras señalan, asimismo, que la situación actual de la TISP no es 
muy diferente de aquella en 2006. El uso de gestos o el intérprete ad hoc o acompañante sigue siendo una de las soluciones más recurrentes en los servicios públicos.

Ana-Isabel Foulquié-Rubio analiza la situación actual de la TISP en la Región de Murcia, basándose para ello en investigaciones realizadas previamente por otros autores de la materia. En esta región, se ha optado principalmente por el sistema de subcontratación de los servicios a empresas externas. En caso de ausencia de dichas empresas se recurre a soluciones ad hoc, con la ayuda de niños, familiares o voluntarios que actúan como intérpretes.

Lurdes Auzmendi, Leire Segura, Marina Aparicio y Cande Cabanillas arrojan luz sobre la situación actual de la TISP en Navarra y el País Vasco. Sus conclusiones indican claramente que, cualitativamente, continúan existiendo graves deficiencias. En la justicia y la policía no se cumplen los artículos de la Directiva 64/2010 de la Ley de Enjuiciamiento Criminal. Los servicios de TISP están adjudicados al licitador más barato, siendo el precio el único criterio de valoración, por lo que se siguen poniendo en peligro los derechos de los usuarios alófonos.

Juan-Miguel Ortega-Herráez y María-Jesús Blasco-Mayor abordan en su artículo la TISP en la Comunidad Valenciana. Para ello, utilizan fuentes oficiales, publicaciones en el ámbito de la TISP y datos cualitativos y cuantitativos provenientes de trabajos fin de máster, trabajos fin de grado y memorias de prácticas externas. El capítulo concluye señalando que la provisión de servicios lingüísticos en Valencia es claramente deficiente o muy mejorable, en el caso de la justicia y la policía, y prácticamente inexistente en el caso de la sanidad pública, afirmándose que las conclusiones obtenidas no difieren mucho de las expuestas en 2006. Según los autores, las administraciones públicas y privadas no han aprendido nada en todos estos años, siendo Valencia el motor del crecimiento turístico en España. En algunos casos se opta por la externalización del servicio de traducción e interpretación, sin prever mecanismos de control de calidad, con el perjuicio que podría tener sobre las personas.

Ruth León-Pinilla cierra el libro con la descripción de la presencia de la TISP en los contextos de asilo y refugio, mediante entrevistas semiestructuradas y grupos de discusión dirigidos a la muestra de su estudio, constituida por 5 entrevistas dirigidas a las personas solicitantes de asilo y refugiadas, proveedores de los servicios y intérpretes. Pretende mostrar "la perspectiva de los agentes implicados en la interpretación en contextos de asilo y refugio respecto a la presencia o no de interpretación en dichos contextos". León-Pinilla afirma que, en España, la interpretación en contextos de asilo y refugio "se hace como se puede", sin tener en cuenta las consecuencias que podría acarrear. Sus dos conclusiones son: 1) los refugiados tienen acceso a la interpretación únicamente en el ámbito policial; 2) el número de intérpretes es reducido en el ámbito sanitario y social, y se recurre a soluciones ad hoc.

En definitiva, no cabe duda que la presente obra es un gran avance para la comunidad académica de la profesión del intérprete en los servicios públicos, dado que describe de forma exhaustiva la situación actual de la provisión de los servicios de TISP en España hasta el año 2016. Constituye un punto de referencia para los investigadores del campo, ya que ofrece datos útiles sobre el progreso de la profesión en el territorio nacional. Finalizada la lectura del libro, Consideramos que queda mucho por hacer para mejorar la profesión de la TISP en España. Es necesario trabajar para alcanzar la profesionalización, el reconocimiento y la acreditación de la TISP, mediante la formación, la adopción de códigos de conducta, así como la difusión de la profesión. Para ello, es menester mejorar la oferta académica de numerosas universidades españolas relativa a la profesión de la TISP. 